\title{
Dinâmica populacional e o perfil de mortalidade no município de Montes Claros (MG)
}

\author{
Population dynamics and the profile of mortality \\ in the municipality of M ontes Claros, M inas Gerais State, Brazil
}

M aryaneOliveira-Campos ${ }^{1}$

M arília Borborema Rodrigues Cerqueira ${ }^{2}$

João Felício Rodrigues $\mathrm{N}$ eto $^{3}$

\footnotetext{
1 Programa de Pós-Graduação em Saúde Pública, Universidade Federal deM inas Gerais. Av. Prof. Alfredo Balena 190, Santa Efigênia. 30130-100 Belo Horizonte MG. oliveiracampos.maryane@ yahoo.com.br

2 Estação de Pesquisa da Escola Técnica deSaúde, Universidade Estadual de M ontes Claros. 3Programa de Pós-Graduação em Ciências daSaúde, Universidade Estadual de M ontes Claros. de M ontes Claros.
}

Abstract Thisstudy is an ecological study of time trend. The objective of this study is to assess the population dynamics as the 1980 Census, 1991 and 2000 , and the profile of mortality in the period between 1996 and 2005, in M ontes Claros, $M$ inas Gerais State. We used the information systems database of Datasus and IBGE. The main results indicate that the elderly group increased from $4.1 \%$ in $1980,5.0 \%$ in 1991 to $6.6 \%$ in 2000 . $\mathrm{O} n$ the proportional mortality by cause, diseases of the circulatory system were the first cause of death, and $23.05 \%$ in 1996 and $28.26 \%$ in 2005, followed by cancer $14.13 \%$ in 1996 and $15.69 \%$ in 2005, external causes of morbidity and mortality $11.87 \%$ in 1996 and $12.74 \%$ in 2005 and infectious and parasitic diseases $8.42 \%$ in 1996 and $5.43 \%$ in 2005 . The profile of mortality followed the population dynamics of the city observed a reduction of infectious diseases and an increase in chronic diseases, consistent with the process of population aging.

Key words Population dynamics, M ortality, Aging, Chronic diseases
Resumo Trata-se deum estudo ecológico de tendência temporal, cujo objetivo é verificar a dinâmica populacional conforme os censos de 1980 , 1991 e 2000 e o perfil de mortalidade no período entre 1996 e2005, em M ontes Claros (M G). U tilizou-se o banco de dados dos sistemas de informação do Datasus e IBGE. Os princi pais resultadosindicam que o grupo deidosos passou de $4,1 \%$ em 1980, 5,0\% em 1991, para 6,6\% em 2000. Sobrea mortalidade proporcional por causa básica, as doenças do aparel ho circulatório foram a primeira causa básica de óbito, sendo 23,05\% em 1996 e 28,26\% em 2005; segui ram-se as neoplasias, $14,13 \%$ em 1996 e $15,69 \%$ em 2005 , causas externas demorbidadeemortalidade, $11,87 \%$ em 1996 e 12,74\% em 2005 e doenças infecciosas e parasitárias, 8,42\% em 1996 e5,43\% em 2005. 0 perfil demortalidadeacompanhou a dinâmica populacional do município, observando redução de doenças infecciosas e um aumento das doenças crônicas, compatível com o processo de envelhecimento populacional.

Palavras-chave Dinâmica populacional, M ortalidade, Envelhecimento, D oenças crônicas 
Introdução

0 acelerado processo de industrialização-urbanização brasileiro teve profundo impacto na dinâmica populacional, cultural esanitária do país. Como resultado da queda da mortalidade e da fecundidade, a população envelheceu. A inserção de novos processos de trabalho determinou mudanças de estilos de vida na população, que vêm sendo muitas vezes estimulados pela globalização de mercados e da comunicação $0^{1,2}$. Entre os anos de 2002 e 2030, o mundo irá sofrer uma mudança considerável na distribuição de óbitos, de grupos etários mais jovens para grupos etários mais avançados e de doenças transmissíveis para doenças não transmissíveis ${ }^{3,4}$.

As doenças crônicas não transmissíveis (doenças do aparelho circulatório, neoplasias e outras) e agravos provocados por fatores externos (acidentes e violências) são chamados de doenças e os agravos não transmissíveis (DANT). As DANT são responsáveis por cerca de dois terços da carga de doenças no Brasil. As doenças cardiovasculares, o câncer, as causas externas e o diabetes representam $55,2 \%$ do total de causas de óbito ${ }^{5-8}$. Por outro lado, o país enfrenta 0 desafio da coexistência de novos e velhos agravos, uma vez que a magnitude das doenças infectoparasitárias continua elevada e ressaltam-se, ainda, as grandes desigual dades regionais ${ }^{9-11}$.

O município de Montes Claros, ao norte de $M$ inas Gerais, embora geográfica e politicamente inserido na região Sudeste, tem indicadores socioeconômicos mais próximos da região Nordeste ${ }^{12}$, mantendo os contrastes emblemáticos daquilo que se convencionou chamar de "dois Brasis". Diante deste contexto, este artigo tem como objetivo verificar o processo da dinâmica populacional e o perfil de mortalidade da população de M ontes Claros (M G), entre 1996 e 2005.

\section{Material e métodos}

Trata-se de um estudo ecológico de tendência temporal. A fonte básica de informações utilizada no presente estudo foram os dados disponíveis nos site do Departamento de Informática do SUS (Datasus) e no Instituto Brasileiro de Geografia e Estatística (IBGE), cujo acesso foi em junho, julho e agosto de 2008.

0 processo de envelhecimento populacional foi estudado com base em análises dos censos demográficos de 1980, 1991 e 200013, calculandose indicadores demográficos como percentual de população residente no município em relação à região N orte deM inas Gerais; distribuição etária proporcional nos grandes grupos populacionais; razões de dependência demográfica para a população residente no município e índice de idosos. Para realizar as análises, as idades foram classificadas com base na divisão populacional em grandes grupos etários: de 0 a 14 anos, 15 a 59 anos e 60 anos ou mais de idade ${ }^{14}$.

Diante disso, a análise da distribuição etária do município nas três últimas datas censitárias (1980, 1991 e2000) foi realizada com basena divisão populacional em grandes grupos etários: de 0 a 14 anos, 15 a 59 anos e 60 anos ou mais de idade.

O limite de idade para a definição de idosos seguiu o parâmetro da Organização Mundial da Saúde (OMS), que estipula a idade de sessenta anos para países em desenvolvimento ${ }^{15}$. 0 índice de envelhecimento representa a proporção de indivíduos com 65 anos ou mais em relação ao total dejovens, deidadeinferior a quinzeanos na população.

As análises do perfil de mortalidade foram realizadas com os dados do Datasus ${ }^{16}$, referentes aos anos de 1996 e 2005. Foi uma opção dos autores estudarem apenas esses dois períodos, por facilidade, pois as causas de morte estão codificadas pela mesma revisão da Classificação I nternacional de doenças (CID).

Para a avaliação da tendência da mortalidade, foram consideradas os seguintes capítulos da CID-10: I (doenças infecciosas e parasitárias DIP); II (neoplasias); IX (doenças do aparelho circulatório) eXX (causas externas de morbidade e mortalidade) ${ }^{17}$.

As informações de mortalidade foram categorizadas segundo a CID-10, calculando-se a mortalidade proporcional por causa básica do óbito em referência ao total de óbitos e as razões de sexo para os capítulos ora listados, para 100.000 habitantes, com os dados disponíveis. Realizou-se, também, análise do perfil demortalidade para os grandes grupos populacionais, proporcionalmente ao total de óbitos para cada grande grupo e para os sexos.

A variação entre os períodos do estudo (1996 e 2005) para grandes grupos e sexo foi calculada, sendo registrada a evolução da mortalidade proporcional considerando-se esses capítulos. Os dados com "idade ignorada" e "sexo ignorado" foram excluídos das análises. 


\section{Resultados}

A cidade sede do estudo possui, atual mente, cerca de 350.000 habitantes, representa o principal pólo regional ea população épredominantemente urbana (apenas 5,8\% da população se concentram na área rural). A população residente no município de M ontes Claros, em 2000, era equivalente a $20,6 \%$ do total populacional da região $\mathrm{N}$ orte de M inas, sendo o maior e mais populoso município da região.

De acordo com a análise da distribuição etária do município, em 1980, o grupo de 0 a 14 anos somava $40,5 \%$ do total da população, passando para 36,8\% em 1991 e 30,0\% em 2000. Os outros grupos de estudo apresentaram movimentos crescentes, observando-se que o grupo de 15 a 59 anos passou de $55,4 \%$ em 1980 para $58,2 \%$ em 1991 e, em 2000,63,4\% do total populacional do município. 0 grupo de idosos, pessoas com sessenta anos ou mais, passou de $4,1 \%$ em 1980 para 5,0\% em 1991 e 6,6\% em 2000 (Gráfico1).

Em relação o grau de dependência total existente entre os indi víduos que constituíam a população montesclarense nos referidos anos, para 0 ano de 1980, a razão de dependência total foi de 75,9\%; em 1991, esta razão somou $66,8 \%$ e, em 2000 , era de $52,2 \%$. Isto significa que, em 2000 , para cada cem pessoas em idade produtiva, existiam aproximadamente 52 pessoas dependentes.

Para as razões de dependência jovem, foram registrados os seguintes valores: $71,3 \%, 61,3 \%$ e 45,6\%, respectivamente, para 1980, 1991 e 2000.

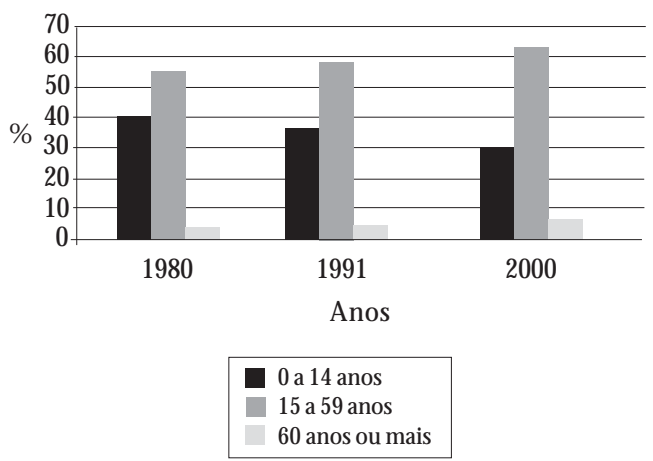

Gráfico 1. População residente dividida por grandes grupos populacionais no município de Montes Claros (MG).

Fonte: IBGE ${ }^{13}$
Por outro lado, as razões de dependência idosa apresentam o aumento relativo dos indivíduos com idade superior a 65 anos. De 4,7\%, em 1980, esta razão passou para 5,5\% em 1991 e, em 2000, totalizou 6,5\%. Em relação ao índice deenvelhecimento, em 1980 era de 6,5\%; em 1991, perfazia aproximados 8,9\% e, em 2001, era de 14,3\%.

A mortalidade proporcional por causa básica (CID - 10 a Revisão) de M ontes Claros (M G) para ambos os sexos, referente aos anos 19962005, mostra a preponderância das doenças do aparel ho circulatório na série de estudo, como a primeira causa básica deóbito, sendo $23,05 \%$ dos óbitos em 1996 e 28,26\% em 2005. Em segundo lugar, encontram-se as neoplasias, com percentuais iguais a 14,13\% em 1996 e 15,69\% em 2005. A terceira causa básica de óbito foram causas externas de morbidade e mortalidade, perfazendo $11,87 \%$ em 1996 e, em 2005, 12,74\% do total dos óbitos. As doenças infecciosas e parasitárias (DIP) encontram-se na quinta posição em ambos os anos, 1996 e 2005, respectivamente, $8,42 \%$ e $5,43 \%$ do total dos óbitos. (Gráfico 2)

0 perfil de mortalidade por razão de sexos para os anos de 1996 e 2005 indica que as causas externas de morbidade e mortalidade obtiveram o resultado expressivo, com 3,59 em 1996 e 5,35 em 2005 - ou seja, para cada cem mulheres que morreram em 1996 e 2005, por esta causa, morreram, respectivamente, 359 e 535 homens. Enquanto as DIP apresentaram 1,68 e 0,74 - passando a registrar um maior número deóbitos de mulheres por DIP, sendo que para cem óbitos de

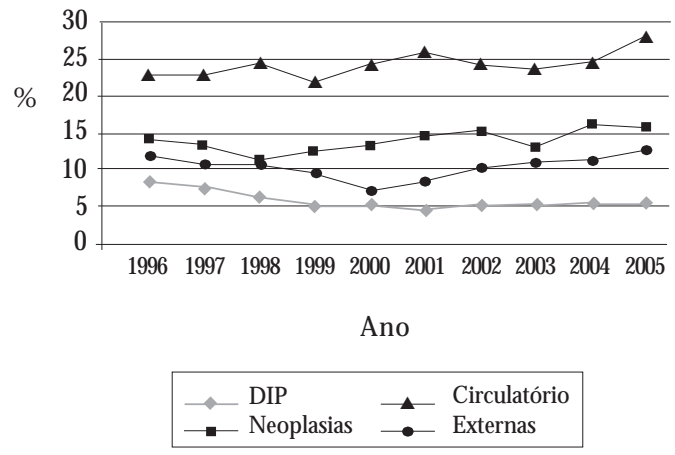

Gráfico 2. M ortalidade por grupos de causa segundo os capítulos da CID 10. M ontes Claros (M G), 1996 a 2005.

Fonte: Datasus ${ }^{16}$. 
mulheres em 2005, morreram 74 homens; as ne oplasias, 1,33 e 1,49 e as doenças do aparelho circulatório, 1,19 e1,21, respectivamente.

A distribuição proporcional deóbitos em relação aos grandes grupos populacionais, entre os anos de 1996 e2005, indicauma queda significativa no grupo de 0 a 14 anos das DIP, decrescendo de 16,12\% em 1996 para 3,57\% em 2005. Observouse movimento decrescente também das doenças do aparelho circulatório, caindo de2,07\%, em 1996, para 0,89\% em 2005. Contrariamente, as neoplasias apresentaram aumento nos anos em questão, de 2,89\% para 5,36\%, assim como para as causas externas de morbidade e mortalidade, crescendo de 8,68\% em 1996 para $12,50 \%$ em 2005.

0 grandegrupo populacional de 15 a 59 anos, respectivo aos anos de 1996 e 2005, apresentou queda nas DIP de 9,42\% em 1996 para 6,74\% em 2005. As demais doenças apresentaram taxas de mortalidade em ascensão, com neoplasias de $13,58 \%$ para $15,16 \%$, doenças do aparelho circulatório de $17,89 \%$ para $20,6 \%$ e causas externas demorbidadeemortalidade de 22,04 para $27,11 \%$, respectivamente.

Para o grupo deindivíduos com sessenta anos ou mais, observou-se redução das DIP, passan- do de $4,88 \%$ para 4,73 e as neoplasias de $18,27 \%$ para $17,31 \%$. Entretanto, a mortalidade por doenças do aparelho circulatório obtivera um aumento de $34,59 \%$ para $37,31 \%$. Observa-se redução das causas externas de morbidade e mortalidade de 3,91\% para 2,69\% (Tabela 1).

A variação do perfil de mortalidade segundo os sexos (masculino/feminino) nos grandes grupos populacionais, para os anos de 1996 e 2005, mostrou que houve um aumento no total de óbitos femininos por DIP para os grupos de 15 a 59 anos e de 60 anos ou mais; como também aumento dos óbitos femininos por doenças do aparelho circulatório, no último grande grupo populacional, de 49,54\%. As neoplasias apresentaram variação crescente para ambos os sexos nos grupos de 15 a 59 anos e 60 anos ou mais, verificando-se uma queda no total de óbitos por esta causa no primeiro grande grupo. Os óbitos masculinos apresentaram variação crescente de 1996 para 2005 por doenças do aparelho circulatório, nos grupos de 15 a 59 anos e 60 anos ou mais, e por causas externas, no grupo de 15 a 59 anos, fato contrário para o grupo feminino, no qual se registrou uma queda de $20,69 \%$, neste mesmo grupo populacional (Tabela 2).

Tabela 1. Distribuição proporcional de óbitos, por Capítulos da CID-10 e anos selecionados, para ambos os sexos e grandes grupos populacionais, M ontes Claros, M inas Gerais, Brasil.

\begin{tabular}{|c|c|c|c|c|c|c|}
\hline \multirow{3}{*}{ Capítulo CID-10 } & \multicolumn{6}{|c|}{ Grandes grupos populacionais e anos selecionados } \\
\hline & \multicolumn{2}{|c|}{0 a 14 anos } & \multicolumn{2}{|c|}{15 a 59 anos } & \multicolumn{2}{|c|}{60 anos ou mais } \\
\hline & 1996 & 2005 & 1996 & 2005 & 1996 & 2005 \\
\hline I - Algumas doenças infecciosas e parasitárias & 16,12 & 3,57 & 9,42 & 6,74 & 4,88 & 4,73 \\
\hline II - Neoplasias & 2,89 & 5,36 & 13,58 & 15,16 & 18,27 & 17,31 \\
\hline IX - Doenças do aparelho circulatório & 2,07 & 0,89 & 17,89 & 20,06 & 34,59 & 37,31 \\
\hline XX - Causas externas de morbidade e mortalidade & 8,68 & 12,50 & 22,04 & 27,11 & 3,91 & 2,69 \\
\hline
\end{tabular}

Fonte: IBGE ${ }^{13}$ eD atasus $^{16}$.

Tabela 2. Taxas de mortalidade por grande grupo populacional em 100.000 habitantes, por algumas causas básicas da CID-10 e anos selecionados. Montes Claros (MG).

\begin{tabular}{|c|c|c|c|c|c|c|c|c|}
\hline \multirow{2}{*}{$\begin{array}{l}\text { Grande grupo } \\
\text { populacional }\end{array}$} & \multicolumn{2}{|c|}{ DIP } & \multicolumn{2}{|c|}{$\begin{array}{l}\text { Doenças do aparelho } \\
\text { circulatório }\end{array}$} & \multicolumn{2}{|c|}{ Causas externas } & \multicolumn{2}{|c|}{ Neoplasias } \\
\hline & 1996 & 2005 & 1996 & 2005 & 1996 & 2005 & 1996 & 2005 \\
\hline 0 a 14 anos & 43,70 & 3,89 & 5,60 & 0,97 & 22,41 & 13,62 & 10,09 & 5,84 \\
\hline 15 a 59 anos & 35,54 & 20,26 & 66,86 & 60,32 & 83,13 & 81,50 & 50,60 & 45,58 \\
\hline 60 anos ou + & 217,68 & 194,33 & $1.542,38$ & $1.532,55$ & 174,14 & 110,41 & 814,73 & 711,07 \\
\hline
\end{tabular}

Fonte: Datasus ${ }^{16}$. 


\section{Discussão}

0 envel hecimento populacional significa mudanças na estrutura etária. H ouve mudanças na composição demográfica do município com o decréscimo da população de 0 a 14 anos e aumento dos outros estratos populacionais. Essas mudanças, em Montes Claros, ocorreram devido ao declínio da fecundidade e ao ganho de longevidade, evidenciando o processo de envelhecimento ${ }^{18}$. Faz-se necessário registrar que, pelo fato do município abrigar escolas de ensino fundamental e médio, além de oito faculdades, está atraindo jovens e adultos de municípios vizinhos e outras regiões do estado, para trabalhar ou estudar, acarretando em um acréscimo do grupo etário de 15 a 59 anos.

o município, assim como o país, encontrase no momento da "janela de oportunidade" ${ }^{19}$, quando o grupo de pessoas em idade ativa e reprodutiva, de 15 a 59 anos, apresenta-se com a maior proporção. Esta situação é denominada de "bônus demográfico" 20 , caracterizado pelo período entre o início do processo de envel hecimento e o momento em que o peso proporcional de idosos se torna maior.

As estimativas indicam um movimento de crescente nas razões de dependência total e jovem, ao contrário da razão de dependência idosa, que está em ritmo ascendente. 0 índice decrescente de razões de dependência demográfica de jovens indica que menos pessoas estão nascendo em M ontes Claros, ou seja, há o declínio da fecundidade das mulheres residentes no município. Entretanto, o índice de dependência de mográfica está aumentando para a população de idosos.

No Brasil, o processo de envelhecimento apre senta-se agudo, dado o declínio rápido da fecundidade, ecom ten dência de convergência dosseus níveis. 0 grupo de pessoas idosas, com sessenta anos ou mais de idade, passou de 6,1\% em 1980 para $8,6 \%$ em 200021, projetando-se para $15 \%$ da população brasileira no ano $2020^{22}$. Essa transição demográfica implica mudanças no perfil de mortalidade da população, não obstante a maior longevidade.

Em relação ao perfil de mortalidade no Brasil, em 1930, as doenças infecciosas respondiam por cerca de $46 \%$ das mortes em capitais brasileiras. A partir deentão, verificou-se redução progressiva; em 2003, essas doenças já responderam por apenas 5\% dessas mortes, aproximadamente ${ }^{11}$. Essas transformações se deram, principalmente, com as seguintes condições: (1) 0 aumen- to da expectativa de vida ao nascer, com o consequente incremento da população idosa; (2) 0 processo acelerado de urbanização e de mudanças socioculturais que respondem, em grande parte, pelo aumento dos acidentes edas violências e (3) a redução da mortalidade precoce, especialmente aquela ligada a doenças infecciosas e parasitárias ${ }^{23,24}$.

Embora as taxas de mortalidade por DIP tenham apresentado queda nos três grandes grupos populacionais, a mortalidade está acima dos níveis brasileiros. M ontes Claros apresenta uma dupla carga de doença - alto índice de doenças crônicas e de doenças infecciosas e parasitárias. As DIP correspondem à quinta causa de óbito do município e a sétima do país em 2005, registrando-se mortalidade proporcional por DIP, para 0 município, em percentuais superiores aos do país em toda a série de estudo, 1996-2005. Esta alta taxa de mortalidade por DIP pode ser justificada pelo fato do município ser uma região endêmica para doença de Chagas e leishmaniose visceral.

No ano de 2002, realizou-se um inquérito canino censitário para o diagnóstico da leishmaniose visceral canina na cidade de M ontes Claros. Os autores relatam que as habitações do município são, em sua maioria, extremamente pobres, com deficiência na coleta de lixo e de saneamento básico, muitas áreas de baixos índices socioeconômicos, a convivência com animais domésticos é bastante elevada, resultando em acúmulo de matéria orgânica, proporcionando condições favoráveis para a ocorrência da transmissão da doença ${ }^{25}$.

A ligação entrea pobreza e o risco de doenças contagiosas tende a receber muito mais atenção queo binômio pobreza versus doenças não transmissíveis. As pessoas integrantes de classes de baixo nível socioeconômico têm muito maischances que os ricos de morrer com uma doença infecciosa ou parasitária, com um risco relativo de 1,9. M as essas pessoas também têm mais possibilidades de morrer por doenças cardiovasculares, com taxas de mortalidade $20 \%$ mais altas (um risco relativo de 1,2) 26,27.

As doenças do aparel ho circulatório, neoplasias e causas externas de morbidade (DANT) obtiveram o resultado expressivo no grupo populacional de 15 a 59 anos - faixa de idade produtiva e reprodutiva. Como observamos na análise da distribuição etária do município, houve um acréscimo do contingente populacional montesclarense maior nessa faixa etária que nos outros extratos, aumentando a taxa de mortalidade por DANT eeste pode ser um retrato da dinâmica populacional. 
As doenças crônicas acometem geralmente a população adulta, com $75 \%$ dos casos ocorrendo entreos 15 e 65 anos, sendo que, acima dos 65 anos, apresentam frequência muito elevada e é comum a concomitância de diversas patologias crônica ${ }^{28}$. Entretanto, na população de sessenta anos ou mais de M ontes Claros, a taxa de mortalidade por neoplasias apresentou uma pequena queda, mas as doenças do aparel ho circulatório aumentaram.

Dessa forma, o aumento da longevidade populacional vem repercutir nas demandas de saúde diante da modificação do perfil de saúde da população. A progressão das doenças crônicas pode ser muito mais rápida pela dificuldade de um diagnóstico precoce e um tratamento adequado, estimando-se que, até 2025, entre 80 a $90 \%$ dos casos ocorrerão em parcelas da população de média e baixa renda familiar. A DANT pode ser um determinante do aprofundamento do baixo nível socioeconômico da família, sendo a maioria incapacitável para o trabalho, inicialmente de maneira temporária, podendo levar a situações de invalidez, resultando em aposentadorias precoces ${ }^{10,28}$.

0 perfil de mortalidade por razão de sexos evidencia que os homens morrem mais por DANT, caracterizan do uma feminização do enveIhecimento. A maior sobrevivência das mulheres chama a atenção no município em 2005; para cada cem óbitos de mulheres, registraram-se 535 óbitos de homens ocasionados por causas externas. Há uma maior proporção de óbitos masculinos também por doenças do aparelho circulatório e neoplasias, da ordem de 121 e 149 óbitos masculinos para cem óbitos femininos por essas causas.

Esses dados repetem resultados encontrados na literatura demográfica referentes à sobremortalidade masculina efeminização do processo de envelhecimento. Háum maior número deóbitos masculinos em quase todos os grupos etários, resultando em um maior contingente de mulheres sobreviventes.

A feminização da velhicetem implicações em termos de políticas públicas, pois uma grande parte das mulheres é viúva, vive só, sem experiência de trabalho no mercado formal e é menos educada. $\mathrm{N}$ em sempre a maior longevidade feminina é vista como vantagem. A maior esperança de vida faz com que muitas mulheres idosas passem pela experiência de debilitação biológica devido a doenças crônicas, enquanto os homens morrem antes ${ }^{29}$. A perspectiva dos gerontólogos é mais otimista quando afirmam que, para as idosas de hoje, a vel hice ea viuvez podem representar um momento de independência erealização ${ }^{30}$.

Em relação a qualidade dos dados do Datasus, as regiões com percentual de causas mal definidas acima de $10 \%$ têm registro considerado inadequa$\mathrm{do}^{31}$. Para os dados de mortalidade do município de M ontes Claros, encontramos $14,6 \%$ dos óbitos enquadrados no grupo de causas mal definidas para 1996 e, para 2005, 12,3\%. A média do período foi igual a $16,6 \%$ do total de óbitos. Estes altos percentuais de causas mal definidas em M ontes Claros indicam uma limitação deste estudo. Vale ressaltar ainda quea mortalidadeproporcional por causas básicas não indica risco de morte por essas causas: indica apenas a contribuição porcentual dessas causas no total do obituário.

De acordo com o Ministério da Saúde ${ }^{32}$, o Sistema de Informações de M ortalidade atingiu, para 2001, 89,3\% de cobertura na região Sudeste, indicando, ainda, a existência de problemas como a subnotificação e subrregistro de óbitos. Entretanto, isso denuncia a necessi dade deintensificar a utilização dessas estatísticas, até como forma de chamar a atenção para a premência de se resolver, com brevidade, os problemas inerentes à qualidade dos dados sobre causas de morte no município.

\section{Considerações finais}

M ontes Claros está, no período, entreo início do processo de envelhecimento e o momento do aumento da proporção de idosos. Este processo pode ser visualizado quando se observa uma redução populacional no grupo de crianças e jovens e um aumento no grupo de adultos e idosos. 0 momento de "janela de oportunidade" pode ser usado para a prevenção de eventuais problemas e/ou questões sociais decorrentes do processo deenvelhecimento.

O perfil de mortalidadeacompanhou a dinâmica populacional do município, passando a uma redução de doenças infecciosas e um aumento das DANT, embora o índice de DIP permaneça alto. 0 declínio de mortalidade concentrou-se entre as doenças infecciosas nos estratos mais jovens da população, que passaram a conviver com os fatores de risco para as doenças crônicas e com a predição a agravos como acidentes e violências. $\mathrm{Na}$ medida em que houve uma ascensão do número de adultos e idosos, as DANT tornaram-se mais frequentes.

O município enfrenta a coexistência denovos e velhos agravos, uma vez que a magnitude das 
DIP continua elevada e há predominância das doenças crônicas e suas complicações, que implicam a maior utilização dos serviços de saúde. Estas mudanças na dinâmica e padrão de distribuição populacional de M ontes Claros alertam para uma adequação do sistema de saúde, que além de cuidar de doenças agudas deve passar a se organizar para dar atenção às DANT.

\section{Colaboradores}

MBR Cerqueira e JFR Neto participaram igualmente de todas as etapas da elaboração do artigo.

\section{Referências}

1. M alta DC, Cezário AC, M orura L, N etto OL, Junior JBS. A construção da vigilância e prevenção das doenças crônicas não transmissíveis no contexto do Sistema Único de Saúde. Epidemiol. Serv. Saúde 2006;15(3):47-65.

2. Lessa I. Doenças crônicas não-transmissíveis no Brasil: um desafio para a complexa tarefa da vigilância. Cien Saude Colet 2004; 9:931-943.

3. Mathers CD, Loncar D. Projections of global mortality and burden of disease from 2002 to 2030. PLOS M ed 2006; 3(11):e442.

4. Lopez AD, Murray CCJL. The global burden of disease, 1990"2020. Nature M ed 1998; 4:1241-1243.

5. Brasil. Ministério da Saúde. Secretaria de Vigilância em Saúde. Saúde Brasil 2004: uma análise da situação de saúde. Brasília: M inistério da Saúde; 2004.

6. Collins JL, Giles HW, Holmes-Chavez A. Old dilemmas, new commitments: toward a 21st century strategy for community health promotion. Prev Chronic Dis [periódico na Internet] [acessado 2007 jul]. Disponível em: http://www.cdc.gov/pcd/issues/2007/ jul/07_0037.htm

7. Organização Pan-Americana da Saúde. Prevenção de doenças crônicas: um investimento vital. Brasília: Organização Pan-Americana da Saúde; 2005.

8. Lopez AD. The evolution of the Global Burden of Disease framework for disease, injury and risk factor quantification: developing the evidence base for national, regional and global public health action. Global Health 2005; 1(5):1-8.

9. Brasil. M inistério da Saúde. Secretaria de Vigilância em Saúde. Departamento de Análise de Situação em Saúde. Saúde Brasil 2006 : uma análise da situação de saúde no Brasil . Braślia: Ministério da Saúde; 2006.

10. Moura de AAG, Carvalho de EF, Silva da NJC. Repercussão das doenças crônicas não-transmissíveis na concessão de benefícios pela previdência social. Cien Saude Colet 2007; 12(6):1661-1672. 
11. Rouquayrol MZ, Almeida Filho N. Doenças e agravos não-transmissíveis: bases epidemiológicas. In: Rouquayrol MZ, Almeida Filho N, organizadores. Epidemiologia \& saúde. 6a ed. Rio de Janeiro: Medsi; 2003.

12. Assessoria para assuntos da Sudene. Área do polígono das secas em M inas Gerais. Belo Horizonte: Palácio dos Despachos; 2000. [M imeo]

13. Instituto Brasileiro de Geografia e Estatística. Censo demográfico: M inas Gerais. 1980, 1991 e 2000. [acessado 2008 jun]. Disponível em: http://www.ibge. gov.br

14. Carvalho JAM, Sawyer DO, Rodrigues RN. Introdução a alguns conceitos básicos e medidas em Demografia. 2ª ed. São Paulo: ABEP; 1998.

15. Tirado M GA. A percepção dos idosos sobre envelhecimento e independência: um estudo qualitativo no município de Belo H orizonte [tese]. Belo H orizonte (M G): Centro de Desenvolvimento e Planejamento Regional, Universidade Federal de M inas Gerais; 2000.

16. Brasil. Ministério da Saúde. Datasus. Informações demográficas e socioeconômicas: população residente. [acesso 2008 jun]. Disponível em: http://tabnet. datasus.gov.br

17. Prata PR. The Epidemiologic Transition in Brazil. Cad Saude Publica 1992; 8(2):168-175.

18. Cerqueira MBR, Rodrigues RN. Envelhecimento populacional: algumas questões. Unimontes Científica 2005; 7(2):73-82.

19. Santana JA. A influência da migração no processo de envelhecimento populacional das regiões de planejamento do estado de M inas Gerais [dissertação]. Belo Horizonte (M G): Centro de Desenvolvimento e Planejamento Regional, Universidade Federal de Minas Gerais; 2002.

20. Wong LLR, Carvalho AJAM. Demographic bonuses and challenges of the Age structural transition in Brazil. In: XXV IU SSP International Population Conference; 2005; Tours, France.

21. Giatti L, Barreto SM. Saúde, trabalho e envelhecimento no Brasil. Cad Saude Publica 2003; 19(3):759771.

22. Camarano AA, Beltrão KI, Araújo HE, Pinto MS. Transformações no padrão etário da mortalidade brasileira em 1979-1994 e no impacto na força de trabaIho. Brasília: IPEA; 1997.

23. Brasil. M inistério da Saúde. Secretaria de Vigilância em Saúde. Departamento de Análise de Situação em Saúde. Saúde Brasil 2006: uma análise da situação de saúde no Brasil. Brasília: Ministério da Saúde; 2006.
24. Moura AAG, Carvalho EF, Silva NJC. Repercussão das doenças crônicas não-transmissíveis na concessão de benefícios pela previdência social. Cien Saude Colet 2007; 12(6):1661-1672.

25. M onteiro EM, Silva JCF, Costa RT, Costa DC, Barata RA, Paula EV, Machado-Coelho GLL, Rocha M F, Fortes-Dias CL, Santos DE. Leishmaniose visceral: estudo de flebotomíneos e infecção canina em Montes Claros, Minas Gerais. Rev. Soc. Bras. Med. Trop. 2005; 38(2):147-152.

26. Banco M undial. Enfrentando o Desafio das Doenças Não Transmissíveis. Relatório No. 32576-BR 2005. Brasília: Banco M undial/U nidade de Gestão do Setor de Desenvolvimento Humano Região da América Latina e do Caribe; 2005.

27. Lessa I, Araújo MJ, Magalhães L, Almeida Filho N, Aquino E, Costa MCR. Simultaneidade de fatores de risco cardiovascular modificáveis na população adulta de Salvador (BA), Brasil. Rev. Panam. Salud Públ. I Pan Am. J. Public Health 2004; 16(2):131-137.

28. Possas $C$. Social ecosystem health: confronting the complexity and emergence of infectious diseases. Cad Saude Publica 2001; 17(1):31-41.

29. Nogales AMV. A mortalidade da população idosa no Brasil. Como vai? População brasileira. Brasília: IPEA; 1998.

30. Debert GG. A reinvenção da velhice: socialização e processos de reprivatização do envel hecimento. São Paulo: Editora da Universidade de São Paulo/FAPESP; 1999.

31. Chackiel J. Studies of causes of death in Latin America current situation and future perspectives. Siena: International Union for the Scientific Study of Population; Institute of Statistics University of Siena; 1986.

32. Brasil. Ministério da Saúde. Coordenação Geral de Informações e Análise Epidemiológica. Análise da Situação de Saúde. Brasília: M inistério da Saúde; 2004.

Artigo apresentado em 30/09/2008

Aprovado em 17/12/2008

Versão final apresentada em 21/01/2009 